\title{
Endoscopic Submucosal Dissection (ESD) is Being Accepted as a New Procedure of Endoscopic Treatment of Early Gastric Cancer
}

Key words: endoscopic mucosal resection, early gastric cancer, insulation-tip diathermic knife

Endoscopic treatment of early gastrointestinal cancers has been shown to be effective in cure of mucosal malignancies without lymph node metastasis. Endoscopic mucosal resection (EMR) designed to remove mucosal lesions by section through the submucosa is the most important technique for endoscopic treatment of early gastric cancers $(1,2)$. According to gastric cancer treatment guidelines issued in March 2001 by the Japanese Gastric Cancer Association (JGCA), EMR should be indicated for patients with small mucosal cancer and no concomitant lymph node metastasis (3). Intestinal type (well and/or moderately differentiated adenocarcinoma and/or papillary carcinoma) mucosal cancers without evidence of ulcer or ulcer scar measuring less than $2 \mathrm{~cm}$ in diameter for the superficially elevated (IIa) type, or less than $1 \mathrm{~cm}$ in diameter for the flat (IIb) and depressed (IIc) types are included in EMR indication. En-bloc resection is preferable because of the risk of residual cancer left behind EMR. En-bloc resection was achieved in $76 \%$ of EMR cases from analysis of 12 major institutions in Japan (4). Although various EMR techniques have been developed for the purpose of removing gastric lesions easier, the guideline indicates that $2 \mathrm{~cm}$ is maximum cancer size of en-bloc resection (4-6). However, gastric mucosal cancers with a diameter of more than $2 \mathrm{~cm}$ with no lymph node metastasis have been detected. For example intestinal mucosal cancers without ulceration and involvement of the lymphatic or venous vessels have practically no risk of lymph node metastasis irrespective of tumor size (7). New technique has been required to expand the indications for EMR in selected situations that have no risks of lymph node metastasis. The recently developed EMR procedure, endoscopic submucosal dissection (ESD), makes en-bloc resection possible for mucosal cancers greater than $2 \mathrm{~cm}$ in diameter (8-12).

In principle EMR consists of three steps; marking, lifting, and cutting. In the marking step, the superficial extent of the lesion is precisely determined using chromoscopy, and the peripheral margin is outlined by electrocoagulation. The next step, lifting, consists of lifting the lesion to render it polypoid by saline injection. The mucosal and submucosal layers are separated from the muscularis propria at this stage. In the final step, cutting, the lesion is resected by electrodiathermy using snare wires. The concept and technique of ESD is markedly different from conventional EMR. The first and second steps of ESD are identical to those of conventional EMR. After saline injection, incision around the lesion is performed using a needle knife or insulation-tip diathermic (IT) knife to separate the lesion from surrounding normal mucosa (8-12). The isolated lesion is exfoliated en-bloc from the gastric mucosa by cutting the submucosal layer below the lesion. ESD has the advantage of permitting enbloc and histological complete resection regardless of lesion size. Also this method has the disadvantage of long performance time and high frequency of complication as well as the needed for a high level of technical skill. The Questionnaire study for gastric ESD showed the performance time in the case of less than $20 \mathrm{~mm}$ in diameter of gastric lesion was more than 60 minutes in many institutions (13). For largesized lesions, performance time increased noticeably. The frequency of complication in ESD procedures was reported at around 5\% for perforations and 25\% for hemorrhage (13). Therefore, ESD has not yet reached the popularity of the conventional EMR techniques. However, due to recent developments of new devices for ESD, such as the IT-knife, hooking knife, electrosurgical snare, Flex knife, and transparent hood, ESD is gradually gaining acceptance among Japanese endoscopists (14).

In this issue of the Journal, Hirasaki et al reported that clinical pathway and standardization of ESD treatment using IT-knife for mucosal gastric cancer had a successful outcome and promoted the quality of care (15).

Since the ESD procedure is absolutely complicated, clinical pathway and standardization of ESD treatment is thought to be difficult to adopt. However, the introduction of ESD protocol reduced the total length of hospitalization and overall healthcare costs. Some patients did not follow this pathway by delaying the start of the diet because of hemorrhage after ESD. The variance rate of $21.7 \%$ in this study was tolerated. This result suggested that ESD become a common popular endoscopic technique for early gastric cancer in near future. A breakthrough such as new devices or good ideas is necessary for a remarkable development of ESD. 
Mototsugu Kato, MD

Division of Endoscopy, Hokkaido University Hospital,

Sapporo 060-8048

\section{References}

1) Tada M, Murakami A, Karita M, Yonai H, Okita K. Endoscopic resection of early gastric cancer. Endoscopy 25: 445-451, 1993

2) Inoue $H$. Treatment of esophageal and gastric tumors. Endoscopy 33 119-125, 2001.

3) Nakajima T. Gastric cancer treatment guidelines in Japan. Gastric cancer 5: 1-5, 2002.

4) Kojima T, Parra-Blanco A, Takahashi H, Fujita R. Outcome of endoscopic mucosal resection for early gastric cancer. Review of the Japanese literature. Gastrointest Endosc 48: 550-555, 1998.

5) Inoue H, Takeshita K, Hori H, Musaoka Y, Yoneshima H, Endo M. Endoscopic mucosal resection with a cap-fitted panendoscope for esophagus, stomach, and colon mucosal lesions. Gastrointest Endosc 39: 58-62, 1993.

6) Inoue $\mathrm{H}$, Tani M, Nagai $\mathrm{K}$, et al, Treatment of esophageal and gastric tumors. Endoscopy 31: 47-55, 1999.

7) Soetikno RM, Gotoda T, Nakanishi Y, Soehendra N. Endoscopic mucosal resection. Gastrointest Endosc 57: 567-579, 2003.

8) Yamamoto H, Yube T, Isoda N, et al. A novel method of endoscopic mucosal resection using sodium hyaluronate. Gastrointest Endosc 50:
251-256, 1999.

9) Ono H, Kondo H, Gotoda $\mathrm{T}$, et al. Endoscopic mucosal resection for treatment of early gastric cancer. Gut 248: 225-229, 2001.

10) Ohkuwa M, Hosokawa K, Boku N, Ohtu A, Tajiri H, Yoshida S. New endoscopic treatment for intramucosal gastric tumors using an insulated-tip diathermic knife. Endoscopy 33: 221-226, 2001.

11) Miyamoto S, Muto M, Hamamoto $Y$, et al. A new technique for endoscopic mucosal resection with an insulated-tip electrosurgical knife improves the completeness of resection of intramucosal gastric neoplasms. Gastrointest Endosc 55: 576-581, 2002.

12) Yamamoto $H$, Kawata $H$, Sunada $K$, et al. Success rate of curative endoscopic mucosal resection with circumferential mucosal incision assisted by submucosal injection of sodium hyaluronate. Gastrointest Endosc 56: 507-512, 2002.

13) Kato M, Shimizu Y, Nakagawa S, Sugiyama T, Asaka M. The results of questionnaire about endoscopic mucosal resection in stomach. Digestive Endoscopy 15 (Suppl.): S2-S7, 2003.

14) Yahagi N, Fujishiro M, Kakushima N, et al. Endoscopic submucosal dissection for early gastric cancer using the tip of an electrosurgical snare (thin type). Digestive Endosc 216: 34-38, 2004.

15) Hirasaki S, Tanimizu M, Moriwaki T, et al. Efficacy of clinical pathway for the management of mucosal gastric carcinoma treated with endoscopic submucosal dissection using an insulation-tip diathermic knife. Intern Med 43: 1120-1125, 2004. 